\title{
L'être-soi et l'être-seul Le problème de la solitude dans la phénoménologie de Michel Henry
}

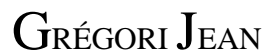

Le projet philosophique le plus général de Michel Henry est d'introduire en philosophie le concept de «subjectivité ». Projet certes paradoxal — la philosophie a-t-elle jamais été autre chose qu'une «philosophie de la subjectivité »? - qu'il ne s'agira ici ni d'expliciter, ni de justifier, mais dont la formulation abrupte nous permettra, à titre de point de départ, de comprendre pourquoi la philosophie henryenne ne semble pas tant «radicaliser» la phénoménologie que revenir en deçà de ses plus fondamentales conquêtes, prêtant le flanc à certaines objections qu'on croyait aussi dépassées que les doctrines à l'encontre desquelles elles avaient été formulées. Et en premier lieu à l'objection de «solipsisme » qui, aussi infondée qu'elle soit par ailleurs, semble néanmoins toucher un trait suffisamment saillant du geste phénoménologique pour que chaque grand phénoménologue - à commencer par le Husserl de la fin de la quatrième des Méditations cartésiennes — feigne de la reprendre à son compte en vue d'en fournir une réfutation argumentée. Et de fait, qu'il s'agisse de la sympathie schelérienne, du Mitsein heideggérien, du regard sartrien ou à fortiori, et dans une tentative de clore définitivement le problème, du visage lévinassien, la phénoménologie semble bien s'être acheminée, depuis l'inscription de l'Autre dans la «mêmeté » d'une subjectivité d'abord autoposée jusqu'à la tentative de lui accorder un absolu primat, vers une forme de 
«désubjectivation» rompant de manière chaque fois plus tranchée avec son supposé «cartésianisme » de départ. Or, le tort de M. Henry serait de suivre le chemin inverse, suffisamment du moins pour que cette objection notoirement obsolète ressurgisse explicitement en 1990 lorsque, dans l'avant-propos de Phénoménologie matérielle et résumant les acquis de sa philosophie — hétérogénéité de la vie et du monde, lien intrinsèque du soi vivant non-mondain et de l'affectivité en tant qu'elle le révèle à lui-même conformément à un autre mode d'apparaître que celui de la phénoménalité « mondaine » — il se trouve contraint de reconnaître :

Dans la venue en soi de la vie en tant que son auto-affection pathétique et comme telle radicalement immanente, prend naissance [...] un Soi dont la matérialité phénoménologique est celle de ce pathos. Un tel «Soi », porté par une subjectivité acosmique, mais aussi bien enfermé en elle, n'est-il pas voué au solipsisme? La possibilité même [...] pour une ipséité pathétique et acosmique d'entrer jamais en relation avec une autre du même type, de s'inscrire dans une intersubjectivité effective et concrète de l'autre, [c'est là l'une des] objections soulevées par les thèses de L'essence de la manifestation dès sa parution et souvent répétées depuis. (Phénoménologie matérielle 8)

Objection soulevée certes, répétée car en apparence fondée, et à vrai dire pour deux raisons : négativement d'abord, parce que la pensée henryenne semble bien s'être déployée, jusqu'au début des années 1990, dans une étrange cécité à la problématique pourtant phénoménologiquement « canonique » de « l'expérience d'autrui ${ }^{1} »$; positivement ensuite, parce que loin de la contourner comme on contournerait une objection à laquelle on ne saurait répondre, c'est tout à fait explicitement et sur un mode thétique que la « solitude » se trouve décrite, dans L'essence de la manifestation, comme un trait essentiel de l'apparaître de la subjectivité immanente, voire comme l'essence même de «l'Essence » — c'est-à-dire du mode d'apparaître qui ne se contente pas de «caractériser» une telle subjectivité, mais avec la «matérialité » duquel, comme subjectivité « vivante », elle se confond. Ainsi dans son $\S 37$, que nous citerons un peu longuement : 
Ce qui a l'expérience de soi, ce qui jouit de soi et n'est rien d'autre que cette pure jouissance de soi-même, que cette pure expérience de soi, c'est la vie. La solitude est l'essence de la vie. Parce qu'elle est l'essence de la vie, la solitude n'est pas un moment de celle-ci, une détermination intervenant dans son histoire qui lui écherait par suite des circonstances ou qu'elle serait susceptible de se donner librement, elle ne résulte pas d'un choix en rapport avec des préoccupations d'ordre moral, d'un impératif. La solitude n'est pas une catégorie de la psychologie ou de l'éthique, mais une catégorie ontologique fondamentale, elle constitue une structure absolument universelle, la structure même de l'essence. Ce qui a l'expérience de soi précisément, ce qui jouit de soi, n'est pas ceci ou cela, c'est l'essence. Car l'essence seule se rapporte originairement à soi, dans cette relation qui la révèle à elle-même dans sa réalité. Mais la révélation à soi de l'essence dans sa relation originaire à soi-même est la Parousie. La Parousie est l'essence de la vie. La Parousie qui est l'essence de la vie, c'est donc là le contenu de la solitude. La solitude est la solitude de l'absolu, mais de l'absolu dans son absoluité, dans la profusion et dans la jouissance de son être propre. (L'essence de la manifestation 354 sq. $)^{2}$

Interroger le problème de la «solitude » dans la phénoménologie de M. Henry — et tenter par ce moyen d'évaluer son apport à cette problématique phénoménologique générale suppose donc que nous parvenions d'abord à répondre à cette double question : d'une part, est-il juste de considérer que la philosophie de M. Henry se soit déployée indépendamment de toute considération relative au problème de «l'expérience d'autrui »?; et d'autre part, et par là-même, la «solitude»dont L'essence de la manifestation fait l'essence de la vie subjective est-elle incompatible avec l'existence et l'expérience d'autrui — de sorte que l'être-soi s'y confonde purement et simplement avec l'être-seul? On le pressent, c'est négativement que nous entendons répondre à ces deux questions, au prix toutefois d'un certain nombre de détours qui constitueront l'essentiel du présent propos.

\section{Solitude et ipséité}

Notre premier détour sera textuel : contrairement à ce que donnent à penser l'œuvre publiée et la littérature secondaire qui lui est consacrée, contrairement à ce que suggèrent également certaines déclarations explicites de M. Henry lui-même ${ }^{3}$, l'édition récente de textes de 
jeunesse inédits sur l'expérience d'autrui — couvrant une période allant approximativement de 1945 à 1950 - établit de manière définitive que, loin qu'il y fût indifférent ou aveugle, le problème de l'expérience d'autrui ne cessa de hanter le jeune M. Henry — né en 1922, il n'avait que vingt-trois ans à la Libération - au point d'accompagner voire de déterminer l'élaboration de certains aspects fondamentaux de sa philosophie ${ }^{4}$. Ainsi de la thèse d'une «duplicité de l'apparaître », conformément à laquelle l'apparaître du monde et des étants intramondains apparaitre tel qu'il compose ce que M. Henry nomme alors, détournant un concept de Scheler, « distance phénoménologique » (L'essence de la manifestation $\S 9)^{5}$ - diffère en nature de l'auto-apparaître affectif de la subjectivité qui le fonde et qui, purement immanent, se caractérise par une immédiateté sans écart du rapport du sujet avec lui-même. Car c'est bien au prisme de cette même duplicité — et comme une contestation de ce qu'il nomme déjà un "monisme ontologique »— que le jeune Henry tente d'élaborer pour lui-même son propre concept de «l'expérience d'autrui » comme expérience «non mondaine », immanente, et à cet égard, selon la terminologie alors employée, «métaphysique »: «Il y a une expérience métaphysique d'autrui ou il n'y a pas d'expérience d'autrui.» (Textes inédits 114) «C'est seulement l'expérience métaphysique d'autrui qui permet de vaincre le solipsisme. Métaphysique signifie ici ceci que cette expérience ne passe pas par la médiation du monde. » (106)

Ainsi se dessine déjà la thèse que tentera d'expliciter, près de trente cinq ans plus tard, Phénoménologie matérielle, celle d'un « rapport» à autrui sans rapport, et dont la perception notamment - qu'elle soit celle de son corps ou, comme chez Scheler, de son psychisme constituerait comme la «condition d'impossibilité ${ }^{6} »$. Thèse audacieuse, que le jeune Henry oppose alors aux théories husserlienne, schelérienne, sartrienne mais aussi heideggérienne de l'«expérience d'autrui »-en tant qu'elles feraient chaque fois de cette expérience une 
expérience «médiate » - mais dont nous ne retiendrons toutefois ici que le mouvement général, et à vrai dire l'aspect paradoxal. Là en effet où il semblait s'agir, pour faire droit à une telle expérience, de ménager au sujet une « sortie » de lui-même — quitte à lui refuser un être propre et à l'assimiler à un être-hors-de-soi, c'est-à-dire finalement à l'ouverture de la phénoménalité du «monde »-, c'est au contraire dans son intériorité radicale, coupée du monde et de ce qui s’y montre, pur et immanent rapport à soi de ce que L'essence de la manifestation nommera «la solitude de l'essence », que le jeune Henry tente de conquérir un rapport à l'autre.

À cet égard, la lecture à laquelle il se livre alors de Sein und Zeit s'avère significative. Commentant, dans une note inédite, son $\S 40$, et le sens du « solipsisme existential » qui, ouvrant «le Dasein comme "solus ipse" », consiste si peu, écrit Heidegger, à transporter « une chosesujet isolée dans le vide indifférent d'une survenance sans-monde qu'il place au contraire le Dasein, en un sens extrême, devant son monde comme monde, et, du même coup, lui-même devant soi-même comme être-au-monde »(Sein und Zeit 187 sq.), le jeune Henry remarque pour lui-même: «Mais ceci ne fait pas sortir de la solitude mais y conduit bien plutôt de façon irrémédiable : le couple sujet-monde est la solitude même; ce qui en fait sortir, c'est l'intériorité, la subjectivité... » (Ms A 4-22-500) ${ }^{7}$

Or, plus paradoxale encore que cette volonté de sortir de la solitude par cette subjectivité qui, saisie comme « intériorité », semblait nous y conduire — plus paradoxale, donc, que cette thèse selon laquelle l'ouverture au monde, et donc aux autres, est précisément ce qui nous condamne au solipsisme — est la nature du reproche adressé ici à Heidegger. Car ce n'est justement pas un excès — dont on comprendrait qu'il nous condamne à la solitude — mais au contraire un déficit d'individuation que le jeune Henry diagnostique dans le Dasein heideggérien, 
dont l'angoisse et l'être-pour-la-mort qui la fonde révélaient pourtant la radicale ipséité et le caractère «insubstituable ». Ainsi dans cette note également inédite :

Contre Heidegger.

Je ne puis mourir pour un autre, mais je ne puis non plus fumer une cigarette, aimer quelqu'un pour un autre. [...] Ma mort me découvre une solitude, mais un amour aussi (aimer c'est, bien souvent, être seul). Donc Heidegger prend [des] déterminations existentielles pour [des] déterminations ontologiques. (Ms A 4-22-2519)

Avant même d'interroger thématiquement le sens de cette opposition entre «déterminations existentielles » et «déterminations ontologiques », il s'agit de prendre cette remarque au sérieux, en dépassant notamment l'impression de trivialité, voire de contresens, qu'elle ne manquera pas de susciter. Car il ne s'est bien entendu jamais agi pour Heidegger de réserver la mienneté du Dasein à son être-pour-la-mort, comme s'il n'était «mien » que dans le « devancement » et la « résolution », demeurant en revanche ontologiquement anonyme et en ce sens «suppléable» dans les autres dimensions de son existence. Bien plutôt la mienneté constitue-t-elle, avec «l'existence », le fondement insuppressible de l'être du Dasein (Sein und Zeit § 9) — sa « représentabilité » ne tenant qu'à la fuite propre à sa quotidienneté, de telle sorte justement que l'être-pour-la-mort, comme projection vers sa possibilité la plus propre, l'arrache au règne $\mathrm{du}$ « On » et le restitue à sa dimension ontologiquement solipsiste, et à cette mienneté que, jusque dans l'extrême « représentabilité » quotidienne, il n'a jamais cessé d'être (239 sq.). Mais c'est ce dont le jeune Henry est bien sûr parfaitement conscient :

La solitude.

Est-elle une détermination ontologique, absolument propre, insurmontable et indépassable, ou seulement une détermination existentielle?

Chez Heidegger elle semble n'être à première vue qu'une détermination existentielle mais en fait, comme elle repose sur un pouvoir-être ontologiquement défini ([1']être-pourla-mort est un existential), c'est une détermination ontologique. (Textes inédits 104). 
Or c'est justement parce que Michel Henry comprend explicitement l'être-pour-la-mort comme une détermination proprement ontologique que la remarque concernant l'impossibilité de fumer ou d'aimer pour un autre s'avère finalement faussement naïve. Car Henry ne se contente pas d'énoncer cette platitude — à laquelle souscrirait évidemment Heidegger — selon laquelle la « représentabilité » n'est jamais une représentabilité « réelle » mais seulement, si l'on ose dire, « représentée », mais bien d'affirmer, et cette fois contre Heidegger, que tout acte subjectif joue ipso facto la fonction que l'analytique existentiale reconnaît à l'être-pour-la-mort, nous renvoyant par conséquent, dans sa structure et son effectivité mêmes, à la solitude vers laquelle seul le devancement résolu était supposé, dans Sein und Zeit, nous ménager un accès. Si, pour paraphraser ce qu'écrit Heidegger du «mourir», nul ne peut prendre son «fumer » ou son « aimer » à autrui, c'est parce que pour Henry tout acte, toute dimension de la subjectivité révèle tout autant, au même titre que le «mourir » et de manière non moins «privilégiée », la solitude existentiale de l'existant. Voilà pourquoi, à la suite de cette note et probablement en la relisant, il ajoute dans une autre couleur: «Que je ne puisse faire quoi que ce soit pour un autre, cela résulte du lien [de l']action et [de la] subjectivité en général à [l']ipséité. » (Ms A 4-22-2519)

Seulement, cette radicalisation du lien entre la subjectivité existante et l'ipséité radicalisation, par conséquent, du « solipsisme existential » de la Fundamentalontologie —, loin de nous permettre de sortir de la solitude - ce qui constituait pourtant le projet explicite du jeune Henry dans son retour à «l'intériorité » - semble davantage encore nous y enfermer. Reformulons en effet la question : comment concilier la condamnation du «couple sujetmonde » en tant que, loin de nous ouvrir à l'altérité, il ne serait que «la solitude même », et le diagnostic, dans l'être-au-monde heideggérien, non pas d'un excès — l'argument serait dès lors limpide — mais bien d'un défaut d'ipséisation, autrement dit de « solitude »? Question d'autant 
plus paradoxale qu'on la répète au prisme du problème de l'intersubjectivité : car si, conformément au réquisit fixé à «l'expérience métaphysique d'autrui » de se produire hors de toute «distance », le problème de la conscience d'autrui «est intimement lié au problème : comment ai-je conscience de moi-même? »(Textes inédits 113); si, plus radicalement, «la connaissance d'autrui » n'est finalement rien d'autre, comme connaissance purement « immanente », que « la connaissance de moi-même » (108), et si la connaissance de moi-même est celle de cette solitude sans monde me reconduisant chaque fois à cette ipséité radicale, force est de constater que cette tentative de « sortir » du solipsisme par ce qui semble au contraire nous y enfermer — l'intériorité, la subjectivité —, revient non pas simplement à y « chercher » une expérience d'autrui, mais bien à l'identifier avec cette expérience « solitaire » non seulement que j'ai de moi-même, mais plus encore que je suis. Exigence abyssale, formulée cette fois par le jeune Henry dans un langage sartrien — «Il n’y aurait véritablement expérience d'autrui que si [était] réalisée l'identité de l'être pour autrui et de l'être pour soi » (111) — et qui nous conduit donc clairement, après celui d'une sortie de la solitude par la solitude elle-même, à l'énoncé d'un second paradoxe. Que signifierait en effet une telle «identité », corrélative de l'absence fondamentale de «distance phénoménologique » revendiquée entre moi et autrui, si ce n'est — objection classique mais qui semble ici atteindre sa portée maximale — que je serais moi-même autrui, et que le problème s'évanouirait dans le mouvement même par lequel on tenterait de le résoudre? Comment un «Soi » défini par sa solitude pourrait-il être «plusieurs », et dans son auto-expérience même, s'ouvrir à l'altérité? Il faudrait qu'autrui et moi ayons, ou plutôt soyons le même Soi, mais que cet être-le-même-Soi — non pas en dépit, mais en vertu même de la solitude de ce Soi — nous ouvre à la pluralité des soi et rende à priori possible l'expérience que nous faisons les uns des autres. 
Or c'est justement à cette exigence que le jeune Henry tente de répondre dans ces notes de jeunesse, convoquant une distinction principielle déjà rencontrée dans les remarques consacrées à Heidegger, mais dont il va s'agir, comme nous l'annoncions alors, de déterminer plus précisément le sens et la portée : «Ma distinction Ontologie-Existence [...] rend seule possible la compréhension des phénomènes fondamentaux qui se manifestent dans l'expérience d'autrui. » (119)

\section{La solitude, entre ontologie et existence}

Certes, c'est d'abord pour s'opposer au modèle d'intersubjectivité appelé par le motif de la «lutte pour la reconnaissance » que plusieurs notes de jeunesse mobilisent cette distinction, afin d'opposer l'attitude que les individus adoptent les uns à l'égard des autres et la manière dont ils se « rapportent» les uns aux autres avant et indépendamment de toute attitude ou «prise de position». Ainsi dans la remarque suivante, consacrée à Sartre mais dont Henry indique clairement la portée plus générale :

Autrui (chez Sartre et peut-être partout ailleurs) n'est connu que lorsqu'il est objet, lorsqu'il est mort : conséquence du monisme. [...]

Mais est-ce pour le connaître ou pour le détruire? De cette façon l'autre est saisi avant, doit être saisi avant pour pouvoir motiver ce projet de connaissance ou de destruction. C'est cette saisie primitive qui est le problème de l'expérience d'autrui. (118)

Et c'est en marge de cette dernière phrase qu'il note au crayon : «[La] dialectique des consciences n’a pas une signification ontologique. » (118)

Ainsi se précise, dans la lignée de nos indications précédentes, le sens de cette distinction, de même que son premier enjeu critique : serait « ontologique » cet être-avec-autrui primitif qui, comme rapport «métaphysique» des ego les uns avec les autres, constituerait non seulement la condition de toute connaissance «mondaine » qu'un individu pourrait acquérir d'un autre 
individu, mais aussi toute «attitude » qu'il pourrait adopter à son égard; au contraire, seraient «existentielles » cette connaissance et ces attitudes elles-mêmes en tant qu'hétérogènes à, quoique fondées sur une telle condition. Or, le propre de la «dialectique des consciences » est justement, comme une conséquence de son «monisme ontologique », de méconnaitre cette distinction, et bien plus, d'ériger l'une des figures «existentielles » possibles que prend, sur le fondement d'un être-ensemble ontologique, la relation à autrui — typiquement, chez Hegel comme chez Sartre, le «conflit» — en un absolu auquel elle assigne le rôle de fondement et la fonction de produire, conformément à une logique se déployant selon ses règles propres, la totalité de ces figures. Seulement, cette manière de jouer la distinction de l'ontologique et de l'existentiel contre le modèle agonistique mobilisé par les théories dialectiques — avec ou sans «synthèse » — de l'intersubjectivité, s'avérerait vite privée de fondement si celle-ci ne se trouvait également mobilisée, comme nous l'annoncions plus haut, à titre de fil directeur d'une réflexion plus profonde et extrêmement originale sur le problème de la solitude, et sur le double paradoxe auquel semble nous acculer le rôle que le jeune Henry entend alors lui conférer : paradoxe d'une sortie de la solitude par sa radicalisation d'une part, paradoxe d'autre part d'une «expérience métaphysique d'autrui » se confondant purement et simplement avec l'expérience de soi.

Et c'est d'ailleurs en contraste avec le sens à première vue tout autre que possède cette différence de l'ontologie et de l'existentiel dans le texte de 1963 que l'originalité de l'usage qu'en proposent ces notes de jeunesse se manifestera le plus clairement : dans L'essence, en effet, elle n'aura plus pour fonction de discriminer, au sein de l'être-avec-autrui, le «métaphysique » et le «mondain », mais de distinguer deux types hétérogènes du rapport à soi de la conscience elle-même — l'adversaire n'y étant alors plus le Hegel de la lutte pour la reconnaissance et de 
l'inégalité des consciences, mais celui de l'inégalité de la conscience avec elle-même, selon qu'il s'agit de la «conscience naturelle» ou de la «conscience philosophique ». Parce que le savoir vrai et le savoir naturel, posera en effet le $\S 18$ de L'essence, ne sont rien d'autres que deux modes de la vie, de l'« existence » de la conscience en général, il s'agit en effet de les opposer à la «structure ontologique » de la conscience elle-même en tant qu'elle s'avère indifférente à cette double modalité dont elle constitue pourtant le fond commun : « La structure ontologique de la conscience est à la fois indifférente et immanente aux déterminations existentielles de celleci. » (L'essence de la manifestation 179) Et c'est dès lors la thèse selon laquelle «l'opposition de la conscience naturelle et du savoir vrai est inessentielle»(179) qui conduit Henry à redéterminer l'«absoluité » du « savoir » comme désignant justement la conscience en tant que telle, une «conscience universelle » qui, dans son indifférence à toutes les modalités du « savoir de soi » — qu'il soit naturel ou philosophique, mondain ou transcendantal —, constitue leur fondement commun et le lieu de leur parousie :

Le savoir absolu désigne l'existence de la conscience dans son essence universelle, non une détermination de cette existence, un mode particulier de sa vie. [...] Parce que la présupposition du savoir vrai de la conscience philosophique et du savoir non vrai de la conscience naturelle est la Parousie, cette présupposition n'est pas un fondement caché derrière la vie de la conscience, elle est la vie consciente elle-même comme telle, la vie de la conscience philosophique comme celle de la conscience naturelle. (174 sq.)

D'où une question à laquelle nous ne pouvons nous soustraire : pourquoi cette distinction de l'ontologique et de l'existentiel, si vigoureusement développée dans L'essence de la manifestation — il ne s'agit de rien de moins que de redéterminer le « savoir absolu » — s'y déploie-t-elle en l'absence de toute considération thématique de l'expérience d'autrui? Pourquoi cette différence principielle, dont le jeune Henry notait qu'elle rend «seule possible la compréhension des phénomènes fondamentaux qui se manifestent dans l'expérience d'autrui » 
(Textes inédits 119), connaît-elle dans L'essence un développement tout à fait indépendant d'une telle problématique? À cette question, une première lecture de L'essence semble permettre de répondre clairement: si le «savoir ontologique» qui s'y trouve circonscrit ne concerne nullement le problème de «l'intersubjectivité », c'est parce qu'il s'agit du mien, de celui de ma conscience ou, plus exactement, c'est parce qu'il n'est rien d'autre que ma conscience elle-même saisie dans son essence. D’où le lien établi au § 52 entre auto-affection et ipséité, nous incitant à voir dans l'immanence et dans son savoir absolu un pur solus ipse; d'où déjà, nous l'avons vu, les développements du $\S 37$ consacrés à la solitude entendue comme «structure interne de l'immanence ». Mais c'est justement cette première impression — selon laquelle cette inscription de la solitude dans la structure ontologique de l'essence donnerait ipso facto congé au problème de l'expérience d'autrui et conduirait à reconnaître la vérité ontologique du solipsisme — que nos développements précédents, sensibles au caractère paradoxal des énoncés henryens, nous ont préparé à rejeter : car ce n'est pas en dépit, mais bien en vertu même de cette solitude de l'essence que le jeune Henry entendait « sortir » du solipsisme, et par là même, nous ménager un accès à autrui. Comment concilier dès lors ces deux lectures de la solitude, l'une nous enfermant dans un solus ipse ontologiquement fondé, l'autre nous en éloignant d'autant plus radicalement que cette solitude sera elle-même saisie dans sa dimension chaque fois plus radicale?

À vrai dire, lors même qu'elles paraissent par ailleurs accentuer, nous l'avons vu, la portée de la solitude, un certain nombre de notes de jeunesse semblent pourtant prendre le contre-pied de l'interprétation ontologique qu'en proposera L'essence de la manifestation, en lui conférant explicitement une dimension purement « existentielle » :

De même que le fait que le monde soit constitué ne signifie pas qu'il est ma représentation (car il se donne précisément comme le contraire de cela), de même le fait que je sois ego cogito, que l'expérience d'autrui ne soit pas possible sans être une 
expérience de cet Ego, loin de rendre l'expérience d'autrui impossible est au contraire ce qui la rend possible. [...] Donc la solitude, pas plus que l'expérience d'autrui, n'est rendue nécessaire par l'essence de l'homme. C'est parce qu'il est ego cogito que cette solitude aussi bien que l'expérience d'autrui sont possibles. (Textes inédits 119) $\mathrm{La}$ solitude n'est pas ontologique mais existentielle...(104)

Mais si, dès lors, «la philosophie de la subjectivité » conduit à reconnaître une vie subjective qui, «existentiellement», peut tout autant prendre la figure de l'«être-avec » que de la «séparation » (108), comment continuer à faire de la solitude l'essence de la subjectivité en tant que telle?

\section{L'être-soi et l'être-seul}

Tout revient, on le pressent, à reposer frontalement cette unique question à laquelle l'œuvre henryenne constitue comme une vaste et lancinante réponse: qu'est-ce que la «subjectivité »? À cet égard, une première note de jeunesse, qui tente justement d'éclairer son sens au prisme du problème de la solitude, nous mettra sur la voie :

La solitude n'est pas liée à la subjectivité : elle est une modalité existentielle de la vie subjective; or même la communication, l'être avec est une modalité existentielle de cette même vie. Le lien solitude-subjectivité ne fait que traduire imparfaitement le lien subjectivité-ipséité. Quant à l'expérience d'autrui, c'est une expérience, elle doit être possible comme expérience, et cela implique transcendance + immanence. (109)

Telle est donc la thèse fondamentale qui ici se dessine : si « le lien solitude-subjectivité ne fait que traduire imparfaitement le lien subjectivité-ipséité », si l'un et l'autre liens, par conséquent, ne se recouvrent pas, c'est d'abord parce que si la subjectivité se trouve ontologiquement liée à l'ipséité, ce n'est pourtant qu'existentiellement, comme l'une des modalités possibles mais non nécessaires de son effectuation, qu'elle se trouve déterminée comme solitude. Or derrière cette distinction entre la dimension ontologique de l'ipséité et la détermination existentielle de la solitude s'en cache une seconde qui, la rendant possible, peut 
seule lui conférer une pleine intelligibilité : celle, précisément, de la subjectivité et de la vie subjective. Car refuser à la solitude une portée ontologique — refuser de la lier intrinsèquement et nécessairement à la subjectivité — c'est refuser d'assimiler la subjectivité avec l'expérience que je peux seul, dans $m a$ «vie subjective », en faire. Seulement, parce que l'expérience que j'en fais et peux en faire, celle qui «peut accompagner toutes mes représentations », n'est justement pas autre chose que moi-même, cela signifie également que la subjectivité-ipséité que je suis, qui se révèle à moi en me révélant à moi-même, qui n'est finalement rien d'autre que ce «moimême ", n'est pourtant pas, ontologiquement, la mienne, là même où me rivant à moi-même, elle constitue l'essence de ma mienneté, et de toute mienneté concevable. Ou encore : parce que l'être-soi n'est pas l'être-seul, la subjectivité-ipséité qui me révèle à moi-même — qui fait de moi, dans cette auto-manifestation, le «même » — n'est pas «la seule », et c'est pourquoi elle peut aussi être celle de l'autre. Non pas qu'elle soit par là même « la sienne », par opposition à la mienne, bien qu'elle soit nôtre, pour autant qu'elle détermine pour chacun de nous ce qui est à chaque fois sien: l'être-soi n'est pas plus ce qui fait que je suis moi que ce qui fait que les autres sont eux-mêmes. Mais c'est dire enfin que l'ipséité située, ontologiquement, au fondement de toute subjectivité - et différant en nature des vies subjectives dont elle constitue pourtant l'ipséité — n'est en elle-même pas plus «plusieurs » que seule, n'est pas plus une que multiple, et c'est ce qu'établit une note de jeunesse à cet égard fondamentale :

Pluralité des ego.

Certes, mais dire [qu'] il y a plusieurs ego, c'est faire apparaître dans le transcendant des conditions du problème qui appartiennent à une tout autre sphère. À une sphère précisément où la multiplicité n'a pas de sens. De même que dire que la conscience de soi est singulière c'est promouvoir une fausse conception de la subjectivité qui vient précisément de ce que cette subjectivité est déplacée et n'est plus examinée dans la sphère ontologique qui est la sienne propre. (110 sq.) 
En même temps dès lors qu'elle révèle le déplacement décisif auquel le jeune Henry soumet le problème phénoménologique classique de l'intersubjectivité, cette note témoigne du sens du projet henryen d'introduire en philosophie, comme nous tentions de le formuler plus haut, le concept même de «subjectivité ». Car cette «subjectivité » qui, si elle se confond avec l'ipséité de toute vie — de sorte qu'il s'agit bien chaque fois d'une vie subjective - , ne s'identifie pas pour autant avec cette vie subjective elle-même, ne saurait plus désigner un étant, une propriété de l'étant, pas même un mode d'être de tel ou tel étant, aussi «privilégié » soit-il, mais une essence phénoménologique, c'est-à-dire, dans le lexique de L'essence de la manifestation, un certaine type d'apparaître. Et c'est justement d'un mode d'apparaître qu'il est illégitime de demander s'il est un ou plusieurs : à titre d'essence de la phénoménalité immanente, le fait d'être soi — la subjectiv-ité comme ipse-ité — n'est pas plus multiple ou unique que peut l'être le fait qu'il y a un monde. Ou plus explicitement encore: qu'il y ait un ou plusieurs mondes, un ou plusieurs soi ne change rien au non-sens de la question de savoir si mondané-ité et ipse-ité sont « unes » ou «plusieurs».

De cette thèse fondamentale - qui signe selon nous, et effectivement, la profonde originalité de la phénoménologie henryenne - il s'agit dès lors de tirer toutes les conclusions, et d'abord concernant le problème de «l'expérience d'autrui ». Car elle permet justement d'y déceler un faux problème: du point de vue de la subjectivité comme «essence» «ontologiquement» par conséquent —, il n'y a pas à proprement d'expérience d'autrui, puisqu'il n'y a ni «moi » ni «autrui » mais le fait même d'être soi, comme forme pure de la phénoménalité immanente et condition de tout moi concevable, par la phénoménalisation de laquelle nous sommes tous deux ce que nous sommes - plusieurs soi, certes, mais une seule ipséité, une seule manière, un seul mode phénoménologique pur d'être donné à soi comme soi. 
Or si «l'expérience métaphysique d'autrui», par laquelle les ego transcendantaux «se touchent », n'est finalement rien d'autre que l'expérience de ce « fait d'être soi » caractéristique de tout « vivre », alors ce qu'est l'autre n'est rien d'autre que ce « fait que je suis moi », fait dont l'épreuve, parce qu'elle constitue le soi de tout moi concevable, est justement celle que fait ou qu'est tout soi possible, le mien comme le sien.

Mais dans quelle mesure — et nous sommes ici renvoyés au premier de nos paradoxes — cette expérience de ou du Soi est-elle dès lors une expérience de la «solitude »? Comment concilier la thèse d'une dimension existentielle de la solitude, qui permettait justement, en déliant l'être-seul de l'être-soi, de faire de l'être-soi un mode d'accès à ce qui constitue le soi des autres pour autant qu'il se confond avec l'expérience de l'être-un-Soi, et celle, propre à L'essence de la manifestation, d'une dimension ontologique de cette même solitude, en tant qu'elle est la solitude de l'«essence » elle-même? Ou encore : comment articuler l'être-ensemble du soi et l'être-Soi de la Solitude? Précisément en maintenant la distinction rigoureuse et radicale établie par Henry entre le sujet donné à lui-même dans et par cette «subjectiv-ité », et l'ipsé-ité qui en qualifie ontologiquement l'essence; et par là même, en distinguant clairement la solitude d'un sujet — détermination existentielle, aussi « inessentielle » à cet égard que tout « être-avec » — et la solitude de la subjectiv-ité entendue comme mode d'apparaitre, comme ce « fait d'être soi » qui fait de tout soi le soi singulier qu'il est irréductiblement sans se confondre avec aucun d'entre eux ${ }^{8}$. Le sens d'une telle solitude — solitude non plus d'un « sujet » mais d'un mode d'apparaître — pour autant que nous ne l'entendions plus, nous avons vu pourquoi, dans son opposition à une quelconque pluralité — le sens de cette solitude, donc, en tant qu'elle échappe à l'alternative de l'un et du multiple, devient dès lors clair : il ne s'agit de rien de moins que de 
marquer l'un-ité intrinsèque de l'essence en tant qu'elle se confond avec cette ipsé-ité ontologiquement saisie. Et c'est ce qu'établit clairement le $\S 37$ de L'essence de la manifestation:

L'essence ne reste pas seule comme une chose quand on a enlevé toutes les autres. Quand plus rien d'autre ne subsiste, l'essence reste seule avec soi. La solitude a un contenu. Ce qui est contenu dans la solitude de l'essence est l'essence elle-même. C'est pour cela que l'essence est solitude, parce que son contenu est constitué par elle. Mais l'essence n'est pas en elle comme un contenu mort, mais comme ce avec quoi elle est liée immédiatement, avec quoi elle a rapport. C'est là ce qui demeure dans la solitude de l'essence, la relation de l'essence avec soi comme constitutive de cette essence même et de sa solitude. La solitude de l'essence se laisse comprendre dès lors dans ce qu'elle est : elle est l'unité de l'essence. [...] Ce qui est impliqué dans cette positivité comme la constituant, c'est la relation de l'essence avec soi, relation telle qu'en elle l'essence jouit de soi, a l'expérience de soi, se révèle à elle-même dans ce qu'elle est, telle qu'elle est. Ce qui a l'expérience de soi, ce qui jouit de soi et n'est rien d'autre que cette pure jouissance de soi-même, que cette pure expérience de soi, c'est la vie. La solitude est l'essence de la vie. (354 sq.)

Une telle «ontologisation» de la solitude dans L'essence de la manifestation, il semble certes difficile de ne pas d'abord l'interpréter comme un «recul» par rapport aux notes de jeunesse qui, posant explicitement sa dimension au contraire «existentielle », se ménageaient l'accès, du moins en principe, à un « être-avec » originaire propre à tout « ego métaphysique », et dont la solitude et la communauté ne seraient que des «modes». Mais c'est pour autant que nous ne sommes pas en mesure de poser la question qui ici seule importe : de qui ou de quoi la « solitude ontologique » de L'essence de la manifestation est-elle une détermination structurelle? Non pas de l'ego, de chacun des ego, des ego que nous sommes et que, chacun de nous, est : comme son titre l'indique assez clairement, ce n'est pas de ce qui constitue tel ou tel ego comme tel ou tel, celui de tel ou tel individu, que traite L'essence, mais de l'essence de l'ego, c'est-à-dire, plus profondément, de l'egoïté de l'essence, autrement dit du lien intrinsèque entre un mode de manifestation et sa dimension essentiellement ipséique. Et c'est précisément ce que vient désigner cette «solitude» à laquelle après avoir, dans ses notes de jeunesse, refusé toute 
dimension ontologique, Henry accorde ici le statut d' « essence de l'essence », comme ce qui fait d'elle l'essence du Soi, l'ipsé-ité de tout ipse - essence qui, comme essence des soi, fonde pour eux la possibilité, pour autant qu'ils en participent, de s'éprouver existentiellement dans la solitude comme dans l'être-avec.

\section{Conclusion.}

\section{Solitude humaine et solitude de l'Absolu : le problème de l'essence de l'homme}

Certes, L'essence de la manifestation n'insiste pas à proprement parler sur cette duplicité, et il faudra attendre C'est moi la vérité et Incarnation pour que la distinction des deux « niveaux » de l'investigation henryenne trouve une formulation explicite : distinction, du point de vue du problème de «l'expérience d'autrui », d'une «phénoménologie de l'incarnation » d'un côté, et d'une «phénoménologie de la chair » de l'autre — la seconde se fondant dans la première pour autant qu'en elle seule, écrira Henry pour la seule et unique fois dans son œuvre publiée, se dessine la «portée métaphysique de l'expérience d'autrui »(Incarnation 297); distinction, au fondement de la première, d'un «sens fort » et d'un «sens faible » du concept d'auto-affection, l'un tourné vers la subjectiv-ité, l'autre vers le sujet qui s'y trouve donné à luimême pour autant qu'il l'effectue ${ }^{9}$. Mais en tout état de cause, et bien plus que d'une rupture ou que d'un «tournant», c'est bien d'un approfondissement — rendu toutefois possible par l'adoption d'un tout autre «modèle » — qu'il s'agit ici. Car de manière générale, que viendrait désigner dans la «trilogie chrétienne » la figure du «premier vivant » et de l' «archi-soi », si ce n'est « l'être-Soi » en tant qu'il n'est ni moi-même ni autrui, ni mon soi ni le sien, mais cette «unique » essence phénoménologique pure intrinsèquement ipséique — ontologiquement «seule» - de laquelle nous participons l'un et l'autre? Que désigne le Christ, si ce n'est cette 
«Ipséité essentielle», ce «Soi primordial» en vertu duquel la vie comme essence phénoménologique, c'est-à-dire comme mode pur d'apparaître intrinsèquement ipséisé, «vient en soi », dessinant ainsi à priori «la multiplicité potentielle et indéfinie de tous les moi possibles », et constituant enfin, parce qu'ils sont «des Soi dans l'Ipséité d'un seul et même Soi $»$, cet « être-avec » qui les « précède toujours »(Incarnation 348 sq.)?

C'est dire que le problème de la solitude permet de reposer à nouveaux frais la question du rapport de L'essence de la manifestation et de la «trilogie chrétienne », et plus encore, celle de l'apport spécifique de cette dernière à l'œuvre henryenne, s'il est vrai que renouant avec certaines de ses intuitions de jeunesse, son originalité ne résiderait justement pas dans l'exhibition de ce rapport de la Vie absolue et d'une Première ipséité — laquelle constituait déjà le thème propre de L'essence — mais dans celle, ontologiquement dérivée et où se lit une inspiration davantage anthropologique, de la manière dont les hommes en «participent » et se trouvent ainsi, à priori, liés les uns aux autres.

Mais bien plus que de rendre simplement possible une toute autre compréhension de l'unité de l'œuvre henryenne et de son «évolution», une telle grille de lecture permettrait également de dessiner le cadre d'un débat essentiel, fermé aussitôt qu'ouvert par le $\S 26$ de L'essence de la manifestation, entre les conceptions henryenne et heideggérienne de l'homme. Car c'est bien à la nécessité de dissocier le projet d'une anthropologie philosophique du primat de la finitude propre à la Fundamentalontologie que conduit la pensée henryenne — ce que révèle la première des notes inédites que nous citions au début de notre étude, pour autant que nous la lisions cette fois dans son intégralité : «Le couple sujet-monde est la solitude même; ce qui en fait sortir, c'est l'intériorité, la subjectivité, dans son rapport interne avec l'absolu. » (Ms A 4-22-500) Et plus explicitement encore, dans une dernière note inédite : 
Ipséité, absolu.

[L']homme [n'est] jamais tout à fait seul; sa solitude n'est qu'apparente, si apparente même que c'est justement au sein d'une telle solitude qu'il peut faire l'expérience de l'absolu qui le contient et le penser. (Ms A 4-20-2378.)

Cet absolu qui le «contient », avec lequel entre en rapport la subjectivité pour autant que, au bout de sa solitude, elle en sort, c'est justement le Soi de tout soi, la Subjectivité absolue comme subjectivité de l'Absolu en tant qu'elle se confond avec son absolue Solitude, et qui fonde celle, « existentielle » — possible mais non nécessaire — , de tout sujet individuel. Faire d'une solitude ontologiquement déterminée l'attribut de l'Absolu lui-même — et non du Dasein reconduit à sa finitude essentielle —, tel serait donc, en dernière instance, le propre du geste henryen, capable de donner un sens — ce qui ne signifie évidemment pas une validité inconditionnelle — au projet d' « introduire en philosophie le concept de subjectivité ».

\section{Notes}

${ }^{1}$ Si l'on excepte le $\S 63$ de L'essence de la manifestation - le problème d'autrui n'étant qu'évoqué, à deux ou trois reprises, dans Philosophie et phénoménologie du corps - et quelques passages des deux tomes du livre consacré à Marx (Marx I, ch. II, p. 105-107 et ch. V, p. 397399; Marx II, ch. VII, p. 118-120 et conclusion, p. 471-473), il faut bien attendre la Phénoménologie matérielle pour voir poindre dans l'œuvre publiée l'exigence de l'affronter en et pour lui-même.

${ }^{2}$ Incarnation reformulera à l'identique l'objection : «Réduire à l'immanence, n'est-ce pas réduire chaque phénomène à son auto-donation et ainsi l'enfermer en lui-même, dresser cette "clôture" qui marque le solipsisme d'une impuissance irrémédiable? Le Soi, lui-même interprété à partir de cette auto-donation, considéré bien plus comme constitué par elle, n'élève-t-il pas luimême les murs de sa propre prison, n'est-il pas en sa propre immanence condamné à une solitude insupportable — à jamais incapable de sortir de lui-même, de s'ouvrir à une altérité sans laquelle aucun autre, aucune expérience de l'autre ne semble possible? » (305)

${ }^{3}$ Comme dans cet entretien, contemporain de l'écriture d'Incarnation, où M. Henry laisse entendre que le problème de l'expérience d'autrui ne fut pour lui, somme toute, qu'une sorte de défi intellectuel : «C'est ainsi que [...] j'ai fait travailler mes présupposés sur la question de l'autre, puisque c'est le point sur lequel phénoménologues et philosophes me semblent avoir 
échoué. J'ai voulu voir, à titre de challenge, si ma phénoménologie de la vie pourrait résoudre ce problème que personne n'a résolu à mon sens. » (« Entretien »121)

${ }^{4}$ Voir sur ce point notre édition critique dans la Revue internationale Michel Henry (71-178), et notre présentation «De l'expérience métaphysique d'autrui à l'intersubjectivité en première personne » (16-70), dont l'examen ici mené de la «solitude » constitue, là même où il lui emprunte certains éléments d'analyse, comme l'autre versant. De manière générale, les textes inédits sur lesquels s'appuie cet article remettent profondément en question l'ensemble des études consacrées à la question du «solipsisme » et de "l'expérience d'autrui » dans la pensée de Michel Henry. Ces dernières en effet, se référant exclusivement à l'œuvre publiée, situent la naissance de cette thématique dans la pensée henryenne au début des années 1990, de telle sorte que, d'une manière ou d'une autre, la question qu'elles posent est la suivante : quelles difficultés propres à sa pensée ont-elles conduit $\mathrm{M}$. Henry à accorder une telle attention au problème d'autrui, et les solutions qu'il avancent sont-elles compatibles - et sinon, en quoi exigent-elles de les amender — avec les thèses fondatrices de L'essence de la manifestation? Dans la mesure où les notes inédites établissent factuellement que les «avancées » des années 1990 ne sont qu'une manière pour Henry de retrouver ses intuitions de jeunesse, la question qu'il s'agit désormais de poser est donc inverse : dans quelle mesure les thèses fondatrices de L'essence de la manifestation sont-elles compatibles avec ces intuitions de jeunesse, et dans quelle mesure surtout elles nous permettent de les lire et de les comprendre autrement - et notamment celles qui ont trait au problème de la solitude?

${ }^{5}$ Pour la version schelérienne de ce concept, $c f$. Scheler, Nature et formes de la sympathie, 42 (n.) et 106.

${ }^{6}$ Sur la critique de la «phénoménologie de la perception appliquée à autrui » et l'idée d'une «impossibilité de la perception» comme «condition de l'être en commun », $c f$. Henry, «Réflexions sur la cinquième Méditation cartésienne » (Phénoménologie matérielle 153 sq.).

${ }^{7}$ Nous remercions le professeur Jean Leclercq, directeur du Fonds Michel Henry à l'Université Catholique de Louvain-la-Neuve, de nous avoir permis de consulter et de citer les manuscrits inédits de Michel Henry. Les ajouts entre crochets [ ] indiquent une intervention ou une coupe de notre part. Pour plus d'informations concernant les manuscrits du Fonds Michel Henry - et notamment le système de classement employé — $c f$. Leclercq, «Editorial »9-14, et notre « De l'expérience métaphysique d'autrui » $18 \mathrm{sq}$.

${ }^{8}$ Que la «subjectiv-ité », comme «mode d'apparaître », constitue irréductiblement et en tant que telle la singularité du « soi » qu'elle fonde et qui l'effectue, c'est ce qui signe l'opposition du jeune Henry aux modèles rationalistes voire «romantiques » classiques d'une expérience d'autrui médiatisée par un « universel » duquel participerait chaque individu en tant qu'individu empirique; nous ne pouvons y insister ici, et renvoyons sur ce point à notre texte «De l'expérience métaphysique d'autrui » (35-39).

${ }^{9} C f$. par exemple sur ce point Henry, C'est moi la vérité 135 sq. 


\section{Ouvrages cités}

Heidegger, Martin, Être et temps, trad. E. Martineau, Alençon, Authentica, 1985.

—, Sein und Zeit, Tübingen, Niemeyer, 1963.

Henry, Michel, Marx, Tome I : Une philosophie de la réalité, Paris, Gallimard, 1976.

—, Marx, Tome II : Une philosophie de l'économie, Paris, Gallimard, 1976.

—, Phénoménologie matérielle, Paris, P.U.F., « Épiméthée », 1990.

—, L’essence de la manifestation, Paris, P.U.F., « Épiméthée », 1990.

_, C'est moi la vérité. Pour une philosophie du christianisme, Paris, Seuil, 1996.

—, Incarnation : une philosophie de la chair, Paris, Seuil, 2000.

—, «Entretien avec Virginie Caruana », Henry, Entretiens, Arles, Sulliver, 2005.

-, Revue internationale Michel Henry, $\mathrm{n}^{\circ} 2$ : Textes inédits sur l'expérience d'autrui, Presses universitaires de Louvain, déc. 2010.

Jean, Grégori, «De l'expérience métaphysique d'autrui à l'intersubjectivité en première personne », Revue internationale Michel Henry, $\mathrm{n}^{\circ} 2$ : Inédits sur l'expérience d'autrui, Presses universitaires de Louvain, déc. 2010, p. 16-70.

Leclercq, Jean, «Éditorial », Revue internationale Michel Henry, ${ }^{\circ} 2$ : Inédits sur l'expérience d'autrui, Presses universitaires de Louvain, déc. 2010, p. 9-14.

Scheler, Max, Nature et formes de la sympathie, trad. M. Lefebvre, Paris, Payot, 1928. 